\title{
Pairings Between Measures and Bounded Functions and Compensated Compactness $\left(^{*}\right)$.
}

\author{
GaBRiEle ANzellotti (Povo, Trento)
}

\begin{abstract}
Summary. - For all vectorfields $\psi \in L^{\infty}\left(\Omega, \boldsymbol{R}^{n}\right)$ whose divergence is in $L^{n}(\Omega)$ and for all vector measures $\mu$ in $\Omega$ whose curl is a measure we define a real valued measure $(\psi, \mu)$ in $\Omega$, that can be considered a suitable generalization of the scalar product of $\psi$ and $\mu$. Several properties of the pairing $(\psi, \mu)$ are then obtained.
\end{abstract}

\section{Introduction.}

The integral of a function $f$ with respect to a Radon measure $\beta$ is defined for instance when $f$ is continuous, or, more generally, when $f$ is $\beta$-measurable and summable; it is also quite clear that the integral $\langle t, \beta\rangle$ eannot be defined for a general Lebesgue-measurable (even if bounded) function $f$. However, we shall see that if $\mu \in M\left(\Omega, \boldsymbol{R}^{n}\right)$ is a $\boldsymbol{R}^{n}$-valued Radon measure on an open set $\Omega \subset \boldsymbol{R}^{n}$ and if $\psi \in L^{\infty}(\Omega$, $\left.\boldsymbol{R}^{n}\right)$, then one can define a real valued measure $(\psi, \mu)$ on $\Omega$, that works nicely as the scalar product of $\psi$ and $\mu$, provided one assumes also that

$$
\operatorname{rot} \mu=\left\{\frac{\partial \mu_{i}}{\partial x_{j}}-\frac{\partial \mu_{j}}{\partial x_{i}}\right\}_{i, j=1, \ldots, n} \text { is a measure in } \Omega
$$

$$
\operatorname{div} \psi \in L^{n}(\Omega) \text {. }
$$

We notice that the hypothesis $(0.1)$ is certainly satisfied in the special case that $\mu=D u$ and $u \in B V(\Omega)$. This special case is the first to be investigated, in sections 1 and 2 . We remark that pairings of this type, between admissible stresses and strains $\sigma, \varepsilon(u)$ in elasto-plasticity, have been already considered in [1], [8], [2].

In section 3 , we define and study the pairing $(\psi, \mu)$ in the general case. Certainly, hypotheses $(0.1),(0.2)$ remind one of compensated compactness, and, in fact, we have also a result (theorem 4.1) that extends to our pairing $(\psi, \mu)$ the result of $\mathrm{MU}$ RAT ([10], theorem 2). Actually, both the proof of theorem 4.1 and the definition of $(\psi, \mu)$ depend on a suitable explicit solution of the equation

$$
\operatorname{rot} z=\lambda
$$

(where $\lambda$ is a given measure) which is obtained as in [10].

(*) Entrata in Redazione il 17 aprile 1983. 
In the appendix we have collected a few approximation and extension results that are needed in the paper.

At the beginning of each section we give an outline of its content.

I would like to thank E. DE GroRgI for his encouragement and for some useful comments on the results of this work.

1. - The pairings $\langle\psi, \mu\rangle_{\partial \Omega},(\psi, D u)$.

It is well known that summability conditions on the divergence of a vector field $\psi$ in $\Omega$ yield trace properties for the normal component of $\psi$ on $\partial \Omega$, for instance compare with $[13],[1],[8]$. In this section (theorem 1.2) we define a function $[\psi \cdot v] \in$ $\in L^{\infty}(\partial \Omega)$ which is associated to any vector field $\psi \in L^{\infty}\left(\Omega, \boldsymbol{R}^{n}\right)$ such that div $\psi$ is a bounded measure in $\Omega$. After that, we define the pairing $(\psi, D u)$, when $\psi$ and $u$ belong to suitable spaces, and we give its first properties. Finally, the expected Green's formula relating $[\psi \cdot v]$ and $(\psi, D u)$ is obtained in theorem 1.9, through lemma 1.8 .

Let $\Omega$ be an open set in $\boldsymbol{R}^{n}, n \geqq 2$, and let $p, q$ be extended real numbers such that $1 \leqq p \leqq n, n /(n-1) \leqq q \leqq+\infty$. We shall consider the following spaces:

$$
\begin{aligned}
& B V(\Omega)_{a}=B V(\Omega) \cap L^{q}(\Omega) \\
& B V(\Omega)_{o}=B V(\Omega) \cap L^{\infty}(\Omega) \cap C^{0}(\Omega) \\
& X(\Omega)_{\infty}=\left\{\psi \in L^{\infty}\left(\Omega, \boldsymbol{R}^{n}\right) \mid \operatorname{div} \psi \in L^{p}(\Omega)\right\} \\
& X(\Omega)_{\mu}=\left\{\psi \in L^{\infty}\left(\Omega, \boldsymbol{R}^{n}\right) \mid \operatorname{div} \psi \text { is a bounded measure in } \Omega\right\} .
\end{aligned}
$$

In the next theorem we define a pairing

$$
\langle\psi, u\rangle_{\partial \Omega}: X(\Omega)_{\mu} \times B V(\Omega)_{c} \rightarrow \boldsymbol{R}
$$

and in the following theorem 1.2 we show that this pairing can be represented as

$$
\langle\psi, u\rangle_{\partial \Omega}=\int_{\partial \Omega} \gamma_{\psi}(x) u(x) d H^{n-1}
$$

where $\gamma_{\psi} \in L^{\infty}(\partial \Omega)$ is a suitable function depending on $\psi$.

THEOREA 1.1. - Assume that $\Omega$ is bounded and that the boundary of $\Omega$ is locally the graph of a Lipschitz function. Denote by $\nu(x)$ the outward unit normal to $\partial \Omega$. Then 
there exists a bilinear map $\langle\psi, u\rangle_{\partial \Omega}: X(\Omega)_{\mu} \times B V(\Omega)_{e} \rightarrow \boldsymbol{R}$ such that

$$
\begin{gathered}
\langle\psi, u\rangle_{\partial \Omega}=\int_{\partial \Omega} u(x) \psi(x) \cdot v(x) d H^{n-1} \quad \text { if } \psi \in C^{1}\left(\bar{\Omega}, \boldsymbol{R}^{n}\right) \\
\left|\langle\psi, u\rangle_{\partial \Omega}\right| \leqq\|\psi\|_{\infty, \Omega} \int_{\partial \Omega}|u(x)| d H^{n-1} \quad \text { for all } \psi, u .
\end{gathered}
$$

Proof. - In order for (1.1) to be satisfied, we are bound to set

$$
\langle\psi, u\rangle_{\partial \Omega}=\int_{\Omega} u \operatorname{div} \psi d x+\int_{\Omega} \psi \cdot D u d x
$$

for all functions $u \in B V(\Omega)_{c} \cap H^{1,1}(\Omega)$ and for all vectors $\psi \in X(\Omega)_{\mu}$. Notice that the last term on the right of (1.3) would not have a defined meaning for general $\psi$, if $D u$ were just a measure. The map $\langle\psi, u\rangle_{\partial \Omega}$ is clearly bilinear, when it is defined.

Now we remark that if $u, v \in B V(\Omega)_{c} \cap H^{1,1}(\Omega)$ and $u=v$ on $\partial \Omega$ then one has

$$
\langle\psi, u\rangle_{\partial \Omega}=\langle\psi, v\rangle_{\hat{o} \Omega} \quad \text { for all } \psi \in X_{\mu}(\Omega)
$$

In fact, by lemma 5.4, one can find a sequence of functions $g_{j} \in C_{0}^{\infty}(\Omega)$ such that, for all $\psi \in X(\Omega)_{\mu}$, one has

$$
\begin{aligned}
\langle\psi, u-v\rangle_{\partial \Omega}=\int_{\Omega}(u-v) \operatorname{div} \psi d x+\int_{\Omega} \psi \cdot D(u-v) d x= & \\
= & \lim _{j \rightarrow \infty}\left\{\int_{\Omega} g_{j} \operatorname{div} \psi d x+\int_{\Omega} \psi \cdot D g_{j} d x\right\}=0 .
\end{aligned}
$$

Now we define $\langle\psi, u\rangle_{\partial \Omega}$ for all $u \in B V(\Omega)_{0}$ by setting

$$
\langle\psi, u\rangle_{\partial \Omega}=\langle\psi, w\rangle_{\partial \Omega}
$$

where $w$ is any function in $B V(\Omega)_{0} \cap H^{1,1}(\Omega)$ such that $w=u$ on $\partial \Omega$. This is a valid definition, in view of the preceding remark and because of the extension lemma 5.5.

To prove estimate (1.2), we take a sequence of functions $u_{j} \in B V(\Omega)_{0} \cap C^{\infty}(\Omega)$ that converge to $u$ as in lemma 5.2 (actually, we do not need property 5.10) and we get

$$
\left|\langle\psi, u\rangle_{\partial \Omega}\right|=\left|\left\langle\psi, u_{j}\right\rangle_{\partial \Omega}\right| \leqq\left|\int_{\Omega} u_{j} \operatorname{div} \psi d x\right|+\|\psi\|_{\infty, \Omega} \int_{\Omega}\left|D u_{j}\right|
$$

for all $\psi$ and for all $j$, hence, taking the limit for $j \rightarrow \infty$ we have

$$
\left|\langle\psi, u\rangle_{\partial \Omega}\right| \leqq\left|\int_{\Omega} u \operatorname{div} \psi d x\right|+\|\psi\|_{\infty, \Omega} \int_{\Omega}|D u|
$$


Now, we take a fixed number $\varepsilon>0$ and we consider a function $w$ as in lemma 0.5 . For such a function we have

$$
\left|\langle\psi, u\rangle_{\partial \Omega}\right|=\left|\langle\psi, w\rangle_{\partial \Omega}\right| \leqq\left\|\underset{\substack{\infty, \Omega \\ \Omega \backslash \Omega_{\varepsilon}}}{\int}|\operatorname{div} \psi|+\right\| \psi \|_{\infty, \Omega}\left(\int_{\partial \Omega}|u| d x+\varepsilon\right)
$$

where $\Omega_{\varepsilon}=\{x \in \Omega \mid \operatorname{dist}(w, \partial \Omega)>\varepsilon\}$ and

$$
\lim _{\varepsilon \rightarrow 0} \int_{\Omega \backslash \Omega_{\varepsilon}}|\operatorname{div} \psi|=0
$$

because div $\psi$ is a measure of bounded total variation in $\Omega$. As $\varepsilon>0$ is arbitrary, estimate (1.3) is proved. q.e.d.

THEOREM 1.2. - Let $\Omega$ be as in theorem 1.1. Then there exists a linear operator $\gamma: X(\Omega)_{\mu} \rightarrow L^{\infty}(\partial \Omega)$ such that

$$
\begin{aligned}
& \left\|\gamma_{\psi}\right\|_{\infty, \partial \Omega} \leqq\|\psi\|_{\infty, \Omega} \\
& \langle\psi, u\rangle_{\partial \Omega}=\int_{\partial \Omega} \gamma_{\psi}(x) u(x) d H^{n-1} \quad \text { for all } u \in B V(\Omega)_{o} \\
& \gamma_{\psi}(x)=\psi(x) \cdot \nu(x) \quad \text { for all } x \in \partial \Omega \text { if } \psi \in C^{1}\left(\bar{\Omega}, \boldsymbol{R}^{n}\right) .
\end{aligned}
$$

The function $\gamma_{\psi}(x)$ is a weakly defined trace on $\partial \Omega$ of the normal component of $\psi$, hence we shall denote $\gamma_{\psi}(x)$ by $[\psi \cdot \nu](x)$.

Proof. - Take a fixed $\psi \in X(\Omega)_{\mu}$ and consider the linear functional $G: L^{\infty}(\partial \Omega) \rightarrow \boldsymbol{R}$ defined by

$$
G(u)=\langle\psi, w\rangle_{\partial \Omega}
$$

where $u \in L^{\infty}(\partial \Omega)$ and $w \in B V(\Omega)_{c}$ is such that $\left.w\right|_{\partial \Omega}=u$. By estimate (1.3) of theorem 1.1 we have

$$
|G(u)| \leqq\left\|\psi_{\| \infty, \Omega}\right\| u \|_{L^{1}(\partial \Omega)}
$$

hence there exists a function $\gamma_{\psi} \in L^{\infty}(\partial \Omega)$ such that

$$
Q(u)=\int_{\partial \Omega} \gamma_{\psi}(x) u(x) d H^{n-1}
$$

and the theorem follows. q.e.d.

Clearly, one has $X(\Omega)_{p} \subset X(\Omega)_{\mu}$ for all $p \geqq 1$ and the trace $[\psi \cdot \nu]$ is defined for all $\psi \in X(\Omega)_{p}$. Our next result is quite natural. 
Proposition 1.3. - Let $\Omega$ be as in theorem 1.1 and let $p, q$ be extended real numbers such that

$$
\begin{array}{ll}
\frac{1}{p}+\frac{1}{q}=1 & \text { if } p>1, \\
q=+\infty & \text { if } p=1 .
\end{array}
$$

Then, for all $\psi \in X(\Omega)_{p}$ and for all $u \in H^{1,1}(\Omega) \cap L^{q}(\Omega)$, one has

$$
\int_{\Omega} u \operatorname{div} \psi d x+\int_{\Omega} \psi \cdot \nabla u d x=\int_{\partial \Omega}[\psi \cdot v](x) u(x) d H^{n-1} .
$$

Proof. - Take a sequence of functions $f_{j} \in C^{\infty}(\bar{\Omega})$ such that

$$
f_{i} \rightarrow u \quad \text { in } \quad H^{1,1}(\Omega) \quad \text { and in } \begin{cases}L^{q}(\Omega) & \text { if } q<+\infty \\ L^{\infty}(\Omega) & \text { weak* if } q=+\infty\end{cases}
$$

Now, formula (1.9) holds for all $j$ with $f_{s}$ at the place of $u$ and, taking the limit for $j \rightarrow \infty$, we get our result, recalling that (1.10) implies $f_{j} \rightarrow u$ in $L^{1}(\partial \Omega)$. q.e.d.

In what follows we shall consider pairs $(\psi, u)$ such that one of the following conditions holds

a) $u \in B V(\Omega)_{q}, \psi \in X(\Omega)_{p} \quad$ and $\quad 1<p \leqq n, \frac{1}{p}+\frac{1}{q}=1$;

b) $u \in B V(\Omega)_{\infty}, \psi \in X(\Omega)_{1}$;

c) $u \in B V(\Omega)_{e}, \psi \in X(\Omega)_{\mu}$.

DeFINITION 1.4. - Let $\psi, u$ be such that one of the conditions (1.11) holds for all open sets $A \subset \subset$ сᄃ $\Omega$. Then we define a linear functional $(\psi, D u): C_{0}^{\infty}(\Omega) \rightarrow \boldsymbol{R}$ as

$$
\langle(\psi, D u), \varphi\rangle=-\int_{\Omega} u \varphi \operatorname{div} \psi d x-\int_{\Omega} u \psi \cdot D \varphi d x .
$$

Compare definition 1.4 and the rest of this section with [8].

THeOrem 1.5. - For all open sets $A \subset \Omega$ and for all functions $\varphi \in C_{0}(A)$, one has

$$
|\langle(\psi, D u), \varphi\rangle| \leqq \sup |\varphi| \cdot\|\psi\|_{\infty, \mathcal{A}} \cdot \int_{\mathcal{A}}|D u|
$$

hence the functional $(\psi, D u)$ is a Radon measure in $\Omega$.

Proof. - Let $u$ be fixed and take a sequence $u_{j} \in C^{\infty}(\Omega)$ that converges to $u$ as in lemma 5.1. Take $\varphi \in C^{\infty}(A)$ and consider an open set $V$ such that $A \supset \supset \supset \operatorname{spt} \varphi$. 
For all $j$ we have then

$$
\left\langle\left(\psi, D u_{j}\right), \varphi\right\rangle|\leqq \sup | \varphi|\cdot| \psi \|_{\infty, A} \cdot \int_{V}\left|D u_{j}\right|
$$

and taking the limit for $j \rightarrow \infty$, we get (1.12). q.e.d.

We shall denote by $|(\psi, D u)|$ the measure total variation of $(\psi, D u)$ and, for every Borel set $B \subset \Omega$, we shall denote by $\int_{B}|(\psi, D u)|, \int_{B}(\psi, D u)$ the values of these measures on $B$.

By theorem 1.5 we get immediately the following corollary.

Corollari 1.6. - The measures $(\psi, D u),|(\psi, D u)|$ are absolutely continuous with respect to the measure $|D u|$ in $\Omega$ and one has

$$
\left|\int_{B}(\psi, D u)\right| \leqq \int_{B}|(\psi, D u)| \leqq\|\psi\|_{\infty, A} \int_{B}|D u|
$$

for all Borel sets $B$ and for all open seis $A$ such that $B \subset A \subset \Omega$.

Moreover, by the Radon-Nicodym theorem, for fixed $\psi$, u, there exists a $\left|D_{u}\right|-$-measurable function

$$
\theta(\psi, D u, x): \Omega \rightarrow \boldsymbol{R}
$$

such that

$$
\begin{aligned}
& \int_{B}(\psi, D u)=\int_{B} \theta(\psi, D u, x)|D u| \quad \text { for all Borel sets } B \subset \Omega \\
& \| \theta\left(\psi, D u, x\left\|_{L^{\infty}(\Omega,|D u|)} \leqq\right\| \psi \|_{\infty, \Omega} .\right.
\end{aligned}
$$

REMark 1.7. - If $E$ is an open set with lipschitz boundary in $\boldsymbol{R}^{n}$, then the characteristic function $u$ of $E$

$$
u(x)= \begin{cases}1 & \text { if } x \in E \\ 0 & \text { if } x \notin E\end{cases}
$$

belongs to the space $B V_{100}\left(\boldsymbol{R}^{n}\right)$ and the measure $(\psi, D u)$ in $\boldsymbol{R}^{n}$ coincides with the measure $\left.[\psi \cdot \nu] H^{n-1}\right|_{\partial E}$.

We shall need the following eontinuity lemma in the proof of theorem 1.9 .

LEMMLA 1.8. - Assume that $u, \psi$ satisfy to one of the conditions (1.11) and let $u_{j} \in C^{\infty}(\Omega) \cap B V(\Omega)$ converge to $u$ as in lemma 5.2 (actually, here we do not need 
(5.10)). Then we have

$$
\int_{\Omega}\left(\psi, D u_{j}\right) \rightarrow \int_{\Omega}(\psi, D u)
$$

Proof. - Take a number $\varepsilon>0$, then take an open set $A$ cC $\Omega$ such that

$$
\int_{\Omega}|D u|<\varepsilon
$$

and let $g \in C_{0}^{\infty}(\Omega)$ be such that $0 \leqq g(x) \leqq 1$ in $\Omega$ and $g(x) \equiv 1$ in $A$. We have then

$$
\begin{aligned}
\left|\int_{\Omega}\left(\psi, D u_{j}\right)-\int_{\Omega}(\psi, D u)\right| & \leqq \\
& \left.\leqq K\left(\psi, D u_{j}\right), g\right\rangle-\langle(\psi, D u), g\rangle\left|+\int_{\Omega}\right|\left(\psi, D u_{j}\right)\left|(1-g)+\int_{\Omega}\right|(\psi, D u) \mid(1-g)
\end{aligned}
$$

where

$$
\begin{gathered}
\lim _{j \rightarrow \infty}\left\langle\left(\psi, D u_{j}\right), g\right\rangle=\langle(\psi, D u), g\rangle \\
\max _{j \rightarrow \infty} \lim _{\Omega}\left|\left(\psi, D u_{j}\right)\right|(1-g) \leqq\|\psi\|_{\infty, \Omega} \max _{j \rightarrow \infty} \lim _{\Omega \backslash \Delta}\left|D u_{j}\right|<\varepsilon\|\psi\|_{\infty, \Omega} \\
\int_{\Omega}|(\psi, D u)|(1-g) \leqq \varepsilon\|\psi\|_{\infty, \Omega}
\end{gathered}
$$

and the lemma is proved, as $\varepsilon$ is arbitrary. q.e.d.

We conclude this section by the expected Green's formula, compare with theorem 3.2 in $[8]$, relating the function $[\psi \cdot \nu]$ and the measure $(\psi, D u)$.

Theorem 1.9. - Let $\Omega$ be a bounded open set with Lipschitz boundary and let $\psi, u$ be such that one of the conditions (1.11) holds, then one has

$$
\int_{\Omega} u \operatorname{div} \psi d x+\int_{\Omega}(\psi, D u)=\int_{\partial \Omega}[\psi \cdot \nu] u d H^{n-1}
$$

Proof. - Take a sequence of functions $u_{j} \in C^{\infty}(\Omega) \cap B V(\Omega)$ that converge to $u$ as in lemma 5.2. Then, by lemma 1.8 and proposition 1.3 , one has

$$
\begin{aligned}
\int_{\Omega} u \operatorname{div} \psi d x+\int_{\Omega}(\psi, D u)=\lim \left\{\int_{\Omega} u_{j} \operatorname{div} \psi d x+\right. & \left.\int\left(\psi, D u_{j}\right)\right\}= \\
& =\lim _{j \rightarrow \infty} \int_{\partial \Omega}[\psi \cdot \nu] u_{j} d H^{n-1}=\int_{\partial \Omega}[\psi \cdot \nu] u d H^{n-1}
\end{aligned}
$$


because

$$
\left.\begin{array}{l}
\int_{\Omega}\left(\psi, D u_{j}\right)=\int_{\Omega} \psi \cdot D u_{j} d x \\
\left.u_{j}\right|_{\partial \Omega}=\left.u\right|_{\delta \Omega}
\end{array}\right\} \text { for all } j \cdot \quad \text { q.e.d. }
$$

2. - Representation of $\theta(\psi, D u, x)$.

In this section we shall be concerned with the problem of whether or not one can write

$$
\theta(\psi, D u, x)=\psi(x) \cdot \frac{D u}{|D u|}(x)
$$

where $(D u /|D u|)(x)$ is the density function of the measure $D u$ with respect to the measure $|D u|$. First, we shall see that the answer is affirmative if $D u \in L_{\mathrm{loc}}^{1}(\Omega)$ or if $\psi \in O^{\circ}(\Omega)$; then we shall see that, in any case, (2.1) holds $|D u|^{a}$-almost everywhere, where $|D u|^{a}$ denotes the absolutely continuous part of the measure $[D u \mid$ with respect to the Lebesgue measure $\mathfrak{L}^{n}$ in $\Omega$. An example shows that, in general, (2.1) does not hold $|D u|^{s}$-almost everywhere (where $|D u|^{s}$ is the singular part of $|D u|$ ), as one is not able to define $\psi(x)|D u|^{s}$-a.e. in $\Omega$. However, even if one does not have a representation formula for $\theta(\psi, D u, x)$ in the singular zone of $|D u|$, the function $\theta(\psi, D u, x)$ still enjoys a few properties (proposition 2.6, 2.7, 2.8) that can be useful. In particular, the results in this section will be used in [3] (compare also with [2]) to get some regularity properties of the vector field $(D u /|D u|)(x)$ when $u$ is a solution to a problem $\int_{\Omega} f(x, D u) \rightarrow \min$ and $f(x, p)$ is asimptotically of linear growth in $p$ for large $|p|$.

For the sake of simplicity, we shall assume throughout this section that $\psi \in X(\Omega)_{n}$ and that $u \in B V(\Omega)$, but it is clear that analogous results can be obtained for pairs $(\psi, u)$ satisfying any one of the conditions (1.11). No assumption is needed in this section on the open set $\Omega \subset \boldsymbol{R}^{n}$.

Here is a continuity result.

Propostiton 2.1. - Assume that

$$
\begin{array}{ll}
\psi_{j} \rightarrow \psi & \text { in } L^{\infty}(A)-\text { weak } \\
\operatorname{div} \psi_{j} \rightarrow \operatorname{div} \psi & \text { in } L^{n}(A)-\text { weak }
\end{array}
$$

for all open sets $A \subset \subset \Omega$; then, for all $u \in B V_{\mathrm{loc}}(\Omega)$, one has

$$
\left(\psi_{j}, D u\right) \rightarrow(\psi, D u)
$$


as measures in $\Omega$, and

$$
\theta\left(\psi_{j}, D u, x\right) \rightarrow \theta(\psi, D u, x)
$$

in $L^{\infty}(A)$-weale for all $A \subset \subset \Omega$. where

Proof. - For all $A \subset \subset \Omega$ and for all $j$ we have $\int_{A}\left|\left(\psi_{j}, D u\right)\right| \leqq\left\|\psi_{j}\right\|_{\infty, A} \cdot \int_{A}|D u|$

$$
\sup _{j \in \mathbb{N}}\left\|\psi_{j}\right\|_{\infty, A}=c(A)<+\infty
$$

because of $(2.2)$, hence it is sufficient to check the weak convergence $(2.4)$ on $C_{0}^{1}(\Omega)$ functions. On the other hand, if $\varphi \in C_{0}^{1}(\Omega)$ one has

$$
\left\langle\left(\psi_{i}, D u\right), \varphi\right\rangle=-\int_{\Omega} u \varphi \operatorname{div} \psi_{j} d x-\int_{\Omega} u \psi_{j} D \varphi d x \rightarrow\langle(\psi, D u), \varphi\rangle
$$

and (2.4) is proved.

To show (2.5) we notice that for all $j$, by corollary 1.6, one has

$$
\left\|\theta\left(\psi_{j}, D u, x\right)\right\|_{L^{\infty}\left(A,\left|D_{u}\right|\right)} \leqq\left\|\psi_{j}\right\|_{\infty, A} \leqq c(A)
$$

hence the convergence (2.5) has to be checked only on $C_{0}^{0}(\Omega)$ functions, where it reduces to (2.4). q.e.d.

We shall need the following simple fact.

LEMMA 2.2. - For every function $\psi \in X(\Omega)_{n}$, there exists a sequence of functions $\psi_{j} \in C^{\infty}(\Omega) \cap L^{\infty}(\Omega)$ such that

$\left\|\psi_{j}\right\|_{\infty, \Omega} \leqq\left\|\psi_{\infty}\right\|_{\Omega} \quad$ for all $j$

$\psi_{j} \rightarrow \psi \quad$ in $L^{\infty}(\Omega)$-weak* and in $L_{100}^{p}(\Omega)$ for $1 \leqq p<+\infty$

$\psi_{j}(x) \rightarrow \psi(x) \quad$ at every Lebesgue point $x$ of $\psi$, and uniformly in any set of uniform continuity for $\psi$.

$\operatorname{div} \psi_{j} \rightarrow \operatorname{div} \psi \quad$ in $L_{1 \mathrm{oc}}^{n}(\Omega)$.

Proof. - Just take a sequence $\left\{\eta_{j}\right\}$ of mollifiers and set $\psi_{j}=\eta_{j} * \tilde{\psi}$, where $\tilde{\psi}$ is defined by

$$
\tilde{\psi}(x)= \begin{cases}\psi(x) & \text { if } x \in \Omega \\ 0 & \text { if } x \notin \Omega . \quad \text { q.e.d. }\end{cases}
$$

Now we give the representation results for $\theta(\psi, D u, x)$. 
Proposimion 2.3. - If $\psi \in X(\Omega)_{n} \cap C^{\circ}(\Omega)$ and $u \in B V(\Omega)$ then one has

$$
\theta(\psi, D u, x)=\psi(x) \cdot \frac{D u}{|D u|}(x), \quad|D u| \text {-a.e. in } \Omega \text {. }
$$

Proof. - Formula (2.6) is equivalent to

$$
\langle(\psi, D u), \varphi\rangle=\int_{\Omega} \varphi \psi D u, \quad \forall \varphi \in C_{0}^{1}(\Omega)
$$

and (2.7) is true by definition if $\psi \in O^{1}(\Omega)$. If $\psi$ is general, we take a sequence $\psi_{;}$ as in lemma 2.2 and, by lemma 2.1 , for all $\varphi \in C_{0}^{1}(\Omega)$, we have

$$
\langle(\psi, D u), \varphi\rangle=\lim _{j \rightarrow \infty}\left\langle\left(\psi_{j}, D u\right), \varphi\right\rangle=\lim _{j \rightarrow \infty} \int_{\Omega} \varphi \psi_{j} D u=\int_{\Omega} \varphi \psi D u
$$

where, in the last step, we have used the fact that $\psi_{\text {; }}$ converges uniformly to $\psi$ on. spt $\varphi$. q.e.d.

If $u \in H^{1,1}(\Omega)$, then, for all $\psi \in X(\Omega)_{n}$ and for all $\varphi \in C_{0}^{1}(\Omega)$ one has

$$
\int_{\Omega} \varphi \psi D u d x=-\int_{\Omega} u \operatorname{div}(\varphi \psi) d x=\langle(\psi, D u), \varphi\rangle
$$

and this implies that

$$
\theta(\psi, D u, x)=\psi(x) \cdot \frac{D u}{|D u|}(x), \quad|D u| \text { - a.e. in } \Omega .
$$

For a general $u \in B V(\Omega)$ one has the following result.

THEOREM 2.4. C If $\psi \in X(\Omega)_{n}$ and $u \in B V(\boldsymbol{R})$, one has

$$
\theta(\psi, D u, x)=\psi(x) \cdot \frac{D u}{|D u|}(x), \quad|D u|^{a}-\text { a.e. in } \Omega .
$$

Proof. - Formula (2.8) is equivalent to

$$
\int_{B} \theta(\psi, D u, x)|D u|^{a}(x) d x=\int_{B} \psi(x) \cdot(D u)^{a}(x) d x
$$

for all Borel $B \subset \Omega$. Let $E^{a}$ and $E^{s}$ be two Borel sets such that $E^{a} \cup E^{s}=\Omega, E^{a} \cap$ $\cap E^{s}=\emptyset, \int_{E^{s}}|D u|^{a}=\int_{\varepsilon^{a}}|D u|^{s}=0$ and let $\varepsilon>0$ be fixed. Then let $K$ be a compact set, with $K \subset E^{s}$, such that

$$
\int_{\mathbb{H}^{2} \backslash K}|D u|^{s}<\varepsilon
$$


and take any compact set $B_{0} \subset E^{a}$. We can find an open set $L$ with regular boundary, such that

$$
B_{0} \subset L \subset \Omega \backslash K, \quad \int_{\Sigma \backslash B_{0}}|D u|<\varepsilon
$$

and, by (2.10) it follows that one has also

$$
\int_{L}|D u|^{3}<\varepsilon
$$

Now, take a sequence $u_{j} \in C^{\infty}(L) \cap B V(L)$ approximating $u$ as in lemma 5.2. By lemma 1.8 and corollary 5.3 we have

$$
\begin{array}{r}
\left|\int_{L} \theta(\psi, D u, x) D u-\int_{L} \psi(x) \cdot(D u)^{a}(x) d x\right|=\lim _{j \rightarrow \infty}\left|\int_{L} \psi(x) \cdot D u_{j}(x) d x-\int_{L} \psi(x) \cdot(D u)^{a}(x) d x\right| \leqq \\
\leqq\|\psi\|_{\infty, L} \lim _{j \rightarrow \infty} \int_{L}\left|D u_{j}-(D u)^{a}\right| \leqq\|\psi\|_{\infty, \Omega} \int_{L}|D u|^{s} \leqq\|\psi\|_{\infty, \Omega} .
\end{array}
$$

On the other hand, we have

$$
\left|\int_{L} \psi \cdot(D u)^{a} d x-\int_{B_{0}} \psi \cdot(D u)^{a} d x\right| \leqq \underset{\substack{L \backslash B_{0} \\ L \backslash,}}{\|}|D u| \leqq \varepsilon\|\psi\|_{\infty, \Omega}
$$

and, by corollary 1.6 , we have also

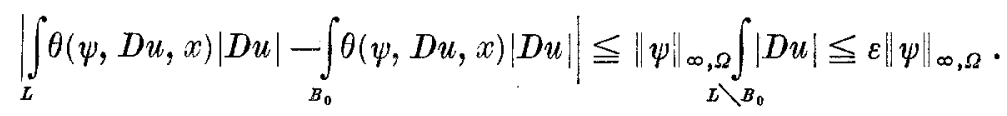

In conclusion we get

$$
\left|\int_{B_{0}} \theta(\psi, D u, x)\right| D u\left|-\int_{B_{0}} \psi \cdot(D u)^{a} d x\right| \leqq 3 \varepsilon\|\psi\|_{\infty, \Omega} .
$$

Hence (2.9) is proved for all compact sets $B \subset E^{a}$. By the regularity properties of Radon measures we have then that (2.9) holds for all Borel sets in $\Omega$. q.e.d.

REMARK 2.5. - If $\psi_{\varrho}(x)=\underset{B_{e}(x)}{f} \psi(y) d y$ is the mean value of $\psi$ in the ball of radius $\varrho$ and center $x$, then we have shown that

$$
\psi_{Q}(x) \cdot \frac{D u}{|D u|}(x) \rightarrow \theta(\psi, D u, x) \quad \text { in } \quad L_{\mathrm{loc}}^{\infty}(\psi,|D u|) \text {-weak* }
$$


where

$$
\left.\begin{array}{l}
\psi_{g}(x) \rightarrow \psi(x) \\
\theta(\psi, D u, x)=\psi(x) \cdot \frac{D u}{|D u|}(x)
\end{array}\right\} \mid D u^{\alpha}-\text { a.e. in } \Omega .
$$

On the other hand, in general, one need not have $\psi_{e}(x) \rightarrow \psi(x)$ in any sense in the zone where $|D u|^{8}$ is concentrated, and the convergence, (2.11) only makes sense. As an example of this situation one can take

$$
\begin{aligned}
& \Omega=\boldsymbol{R}^{2}, \quad E=\left\{x \in \boldsymbol{R}^{2} \mid x_{2}<0\right\}, \quad u(x)= \begin{cases}1 & \text { if } x \in E \\
0 & \text { if } x \in \boldsymbol{R}^{2} \backslash E\end{cases} \\
& \psi=\left(\psi_{1}, \psi_{2}\right), \quad \psi_{1}\left(x_{1}, x_{2}\right)=\operatorname{sen} \frac{1}{x_{2}}, \quad \psi_{2}=0
\end{aligned}
$$

and it is easily seen that $\psi \in L^{\infty}\left(\Omega, \boldsymbol{R}^{2}\right)$, div $\psi=0, \theta(\psi, D u, x)=[\psi \cdot v](x)$ on $\partial E$ (where $v$ is the normal to $\partial E$ ), while the mean values $\psi_{e}(x)$ do not converge on $\partial E$.

Even though the function $\theta(\psi, D u, x)$ cannot be represented in terms of a well

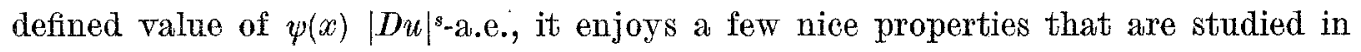
the rest of this section.

Proposition 2.6. - If $\psi \in X(\Omega)_{n}$ and $u \in B V(\Omega)$, then one has

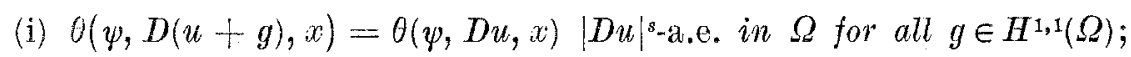

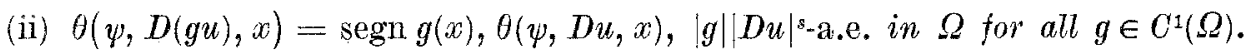

Proof. - (i) Recall that if $D g \in L^{\mathfrak{1}}(\Omega)$ then one has $(D(u+g))^{s}=(D u)^{s}$, then notice that

$$
(\psi, D(u+g))=\theta(\psi, D(u+g), x)|D(u+g)|^{s}+\theta(\psi, D(u+g), x)|D(u+g)|^{a}
$$

while, on the other hand

$$
\begin{aligned}
(\psi, D(u+g))=(\psi, D u)+(\psi, D g) & = \\
& =\theta(\psi, D u, x)|D u|^{s}+\theta(\psi, D u, x)|D u|^{\alpha}+\psi(x) \cdot D g(x) .
\end{aligned}
$$

Equating the two expressions for the singular part of $(\psi, D(u+g))$ we get

$$
\theta(\psi, D(u+g), x)|D u|^{\prime s}=\theta(\psi, D u, x)|D u|^{s}
$$

and (i) follows.

(ii) For all test functions $\varphi \in C_{0}^{1}(\Omega)$ we have

$$
\langle(\psi, D(g u)), \varphi\rangle=\langle(\psi, D u), g \varphi\rangle+\int_{\Omega}(\psi \cdot D g) u \varphi d x
$$


hence we have, for all Borel sets $B \subset \Omega$,

$$
\begin{aligned}
\int_{B} \theta(\psi, D(g u), x)|D(g u)|=\int_{B} \theta(\psi, D u, x) g|D u|^{s}+ & \\
& +\int_{B} \theta(\psi, D u, x) g|D u|^{a}+\int_{B} \psi \cdot D g u d x .
\end{aligned}
$$

Recalling that $|D(g u)|^{s}=|g||D u|^{s}$ and equating the singular parts on the two sides of (2.12) we get (ii). q.e.d.

For all functions $u: \Omega \rightarrow \boldsymbol{R}$ let us consider the sets

$$
E_{u, t}=\{x \in \Omega \mid u(x)>t\}
$$

If $u \in B V(\Omega)$, it is well known [9], [5] that the characteristic functions

$$
\chi_{u, t}(x)= \begin{cases}1 & \text { if } x \in E_{u, t} \\ 0 & \text { if } x \notin E_{u, t}\end{cases}
$$
of the sets $E_{u, t}$ are in $B V(\Omega)$ for $\mathcal{L}^{1}$-almost all $t \in \boldsymbol{R}$; moreover, the function $t \mapsto \int_{\Omega}\left|D \chi_{u, t}\right|$
is $\mathcal{L}^{1}$-measurable and the coarea formula

$$
\int_{\Omega} f(x)|D u|=\int_{-\infty}^{+\infty} d t \int_{\Omega} f(x)\left|D \chi_{\chi_{u}, t}\right|
$$

holds for every $|D u|$-summable function $f: \Omega \rightarrow \boldsymbol{R}$. It follows that a set $B \subset \Omega$ has $|D u|$-measure zero if and only if for $\mathcal{L}^{1}$-almost all $t \in \boldsymbol{R}$ one has $\int_{B}\left|D_{\chi_{u, t}}\right|=0$. For
later use we recall also that one has

$$
\frac{D u}{|D u|}(x)=\frac{D \chi_{u, t}}{\left|D \chi_{u, t}\right|}(x), \quad\left|D \chi_{u, t}\right|-\text { a.e. in } \Omega
$$

for $\mathfrak{L}^{1}$-almost all $t \in \boldsymbol{R}$.

Now we shall give a "slicing 》 result that links the measure $(\psi, D w)$ with the measures $\left(\psi, D \chi_{u, t}\right)$.

Proposition 2.7. - If $\psi \in X(\Omega)_{n}$ and $u \in B V(\Omega)$, then we have:

(i) for all functions $\varphi \in C_{0}^{0}(\Omega)$, the function $t \mapsto\left\langle\left(\psi, D \chi_{u, t}\right), \varphi\right\rangle$ is $\mathcal{L}^{1}$-measurable and

$$
\langle(\psi, D u), \varphi\rangle=\int_{-\infty}^{+\infty}\left\langle\left(\psi, D_{\chi u, t}\right), \varphi\right\rangle d t
$$


(ii) for all Borel sets $B \subset \Omega$, the function $t \rightarrow \int_{B}\left(\psi, D_{\chi_{u, t}}\right)$ is $\mathfrak{L}^{1-m e a s u r a b l e ~ a n d ~}$ $\int_{B}(\psi, D u)=\int_{-\infty}^{+\infty} d t \int_{B}\left(\psi, D \chi_{u, t}\right) ;$

(iii) $\theta(\psi, D u, x)=\theta\left(\psi, D \chi_{u, t}, x\right)\left|D \chi_{u, t}\right|$-a.e. in $\Omega$ for $\mathcal{L}^{1}$-almost all $t \in \boldsymbol{R}$.

Proof. - (i) Take a sequence of functions $\psi_{j} \in C^{\infty}(\Omega) \cap L^{\infty}(\Omega)$ that converge to $\psi$ as in lemma 2.2. Then, for all $j$, we have, by the coarea formula,

$$
\begin{aligned}
\left\langle\left(\psi_{i}, D u\right), \varphi\right\rangle=\int_{\Omega} \psi_{j}(x) & \cdot \frac{D u}{|D u|}(x) \varphi(x)|D u|= \\
& =\int_{-\infty}^{+\infty} d t \int_{\Omega} \psi_{j}(x) \cdot \frac{D \chi_{u, t}}{\left|D \chi_{u, t}\right|}(x) \varphi(x)\left|D_{\chi_{u, t} \mid}\right|=\int_{-\infty}^{+\infty}\left\langle\left(\psi_{j}, D_{\chi_{u, t}}\right), \varphi\right\rangle d t
\end{aligned}
$$

where

$$
\left|\left\langle\left(\psi_{i}, D \chi_{u, t}\right), \varphi\right\rangle\right| \leqq\left\|\psi_{\infty, \Omega}\right\| \varphi \|_{\infty, \Omega} \int_{\Omega}\left|D \chi_{u, t}\right|
$$

Recalling proposition 2.1, taking the limit in (2.14) for $j \rightarrow \infty$, by the dominated convergence theorem we get the proof of (i).

We shall prove (ii) after (iii). Let's prove (iii). Take $a, b \in \boldsymbol{R}$ and consider the function $v \in B V(\Omega)$ defined by

$$
v(x)= \begin{cases}b & \text { if } b \leqq u(x) \\ u(x) & \text { if } a \leqq u(x) \leqq b \\ a & \text { if } u(x) \leqq a\end{cases}
$$

then we have $E_{u, t}=E_{v, t}$ for all $t$ such that $a \leqq t<b$, hence

$$
\begin{aligned}
& \left.\begin{array}{l}
D_{\chi_{u, t}}=D_{\chi_{v, t}} \\
\frac{D_{\chi_{u, t}}}{\left|D_{\chi_{u, t} \mid}\right|}(x)=\frac{D \chi_{v, t}}{\left|D_{\chi_{v, t} \mid}\right|}(x)
\end{array}\right\} \text { if } a \leqq t<b \\
& D_{\chi_{v, t}}=0 \quad \text { if } t \geqq b \text {, or } t<a
\end{aligned}
$$

and it follows that

$$
\begin{aligned}
\frac{D u}{|D u|}(x)=\frac{D \chi_{u, t}}{\left|D_{\chi_{u, t}}\right|}(x)= & \frac{D \chi_{v, t}}{\left|D_{\chi_{v, t}}\right|}(x)=\frac{D v}{|\overline{D v}|}(x), \\
& \left|D_{\chi_{v, t} \mid}\right|-\text { a.e. in } Q \text { for } \mathcal{L}^{1-a l m o s t} \text { all } t \in \boldsymbol{R}
\end{aligned}
$$


that is

$$
\frac{D u}{|D u|}(x)=\frac{D v}{|D v|}(x), \quad|D v|-\text { a.e. in } \Omega
$$

Now it follows that, for every $\psi \in X(\Omega)$ we have

$$
\theta(\psi, D u, x)=\theta(\psi, D v, x), \quad|D v| \text {-a.e. in } \Omega \text {. }
$$

In fact, if $\psi_{j} \rightarrow \psi$ as in lemma 2.2 , we have, for all $j$

$$
\theta\left(\psi_{j}, D u, x\right)=\psi_{j}(x) \cdot \frac{D u}{|D u|}(x)=\theta\left(\psi_{j}, D v, x\right), \quad|D v|-\text { a.e. in } \Omega
$$

and taking the limit for $j \rightarrow \infty$, by the uniqueness of the limit in the $L^{\infty}(\Omega,|D v|)$ weak* topology, we get (2.15). Finally, using statement (i) for $v(x)$, we have, for all $a<b$ and for a fixed $\varphi \in C_{0}^{\infty}(\Omega)$,

$$
\langle(\psi, D v), \varphi\rangle=\int_{-\infty}^{+\infty}\langle(\psi, D \chi,, t), \varphi\rangle d t
$$

i.e., by the coarea formula and (2.15):

$$
\int_{a}^{b} d t \int_{\Omega} \theta(\psi, D u, x) \varphi(x)\left|D \chi_{v, t}\right|=\int_{a}^{b} d t \int_{\Omega} \theta\left(\psi, D \chi_{v, t}, x\right) \varphi(x)\left|D \chi_{v, t}\right|
$$

and this implies that

$$
\int_{\Omega} \theta(\psi, D u, x) \varphi(x)\left|D_{\chi_{u, t}}\right|=\int_{\Omega} \theta\left(\psi, D \chi_{u, t}, x\right) \varphi(x)\left|D \chi_{u, t}\right|
$$

for ${ }^{1}$-almost all $t \in \boldsymbol{R}$. If $S$ is a countable dense set in $C_{0}^{\infty}(\Omega)$ with respect to the uniform convergence, it is possible to find a set $N \subset \boldsymbol{R}$ such that $\mathfrak{L}^{1}(N)=0$ and that (2.16) holds for all $t \in \boldsymbol{R} \backslash N$ and for all $\varphi \in S$. It follows that for all $t \in \boldsymbol{R} \backslash N$ one has

$$
\theta(\psi, D u, x)=\theta\left(\psi, D \chi_{u, t}, x\right)
$$

as wanted.

To prove (ii) we notice that, by (iii), we have

$$
\begin{aligned}
\int_{B}(\psi, D u)=\int_{B} \theta(\psi, D u, x)|D u|= & \int_{-\infty}^{+\infty} d t \int_{B} \theta(\psi, D u, x)\left|D \chi_{u, t}\right|= \\
& =\int_{-\infty}^{+\infty} d t \iint_{B} \theta\left(\psi, D \chi_{u, t}, x\right)\left|D \chi_{u, t}\right|=\int_{-\infty}^{+\infty} d t \int\left(\psi, D \chi_{u, t}\right) . \quad \text { q.e.d. }
\end{aligned}
$$


Our next result is a consequence of proposition 2.7 .

Proposimion 2.8. - If $\alpha: \boldsymbol{R} \rightarrow \boldsymbol{R}$ is an increasing function of class $C^{1}$, then one has

$$
\theta(\psi, D(\alpha \circ u), x)=\theta(\psi, D u, x), \quad|D u| \text {-a.e. in } \Omega
$$

where $(x \circ u)(x)=\alpha(u(x))$.

Proof. - First, notice that

$$
E_{u, t}=\{x \in \Omega \mid u(x)>t\}=\{x \in \Omega \mid(\alpha \circ u)(x)>\alpha(t)\}=E_{\alpha, u, \alpha(t)}
$$

so that, for almost all $t \in \boldsymbol{R}$, one has

$$
D \chi_{u, t}=D \chi_{\alpha \circ u, x(t)}
$$

hence, for almost all $t \in \boldsymbol{R}$ one has also

$$
\theta(\psi, D u, x)=\theta\left(\psi, D_{\chi_{u, l}}, x\right)=\theta\left(\psi, D_{\chi_{x} \circ u, \alpha(t)} x\right)=\theta(\psi, D(\propto \circ u), x)
$$

$\left|D \chi_{u, b}\right|$-a.e. in $\Omega$, and (2.17) follows. q.e.d.

\section{3. - The pairing $\langle\psi, \mu\rangle$.}

In this section we define a pairing $(\psi, \mu)$ when $\psi \in X(\Omega)_{n}$ and $\mu$ is a measure whose curl is also a measure. The key lemma is lemma 3.4 ; the idea for solving the equation rot $z=\lambda$ is the same as in [10], but we cannot use Rellich theorem to show the compactness of the operator $Z: \lambda \rightarrow z$, as we do not have sufficient information on the derivatives of $Z(\lambda)$, and we use instead the information on the translations of $Z(\lambda)$.

The pairing $(\psi, \mu)$ is then defined, when $\mu$ has a compact support, noticing that one can write $\mu=f+D u$, where $f \in L_{1 \mathrm{loc}}^{1}\left(\boldsymbol{R}^{n}\right)$, $\operatorname{rot} f=\operatorname{rot} \mu, u \in B V_{\mathrm{loc}}\left(\boldsymbol{R}^{n}\right)$, and using the results of section 1 . When $\mu$ does not have a compact support we localize and then we glue together the pieces. The results of section 1 and 2 are then used to derive a few properties of the pairing $(\psi, \mu)$, that are collected in theorem 3.8 .

We shall denote by $\boldsymbol{M}\left(\Omega, \boldsymbol{R}^{N}\right)$ the space of the $\boldsymbol{R}^{N}$ valued Radon measures in $\Omega$. We shall set $M_{0}\left(\Omega, \boldsymbol{R}^{N}\right)=\left\{\mu \in M\left(\Omega, \boldsymbol{R}^{N}\right)\right.$ such that spt $\mu$ is compact $\}$ and we shall write simply $M(\Omega)$ instead of $M(\Omega, \boldsymbol{R})$.

We shall use the following well known facts, that we recall for convenience. 
FACT 3.1. - If $f \in L_{100}^{1}\left(\boldsymbol{R}^{n}\right)$ and $\mu \in M_{0}\left(\boldsymbol{R}^{n}\right)$, then the convolution $f * \mu$ is in $L_{100}^{1}\left(\boldsymbol{R}^{n}\right)$ and

$$
\int_{\mathcal{A}}|f * \mu| \leqq \int_{A-s p t \mu}|f| \cdot\|\mu\|
$$

where $\|\mu\|=\int_{\mathbb{R}^{n}}|\mu|$ and $A-\operatorname{spt} \mu=\{x-y \mid x \in A, y \in \operatorname{spt} \mu\}$.

FACT 3.2. (Compactness criterion.) - Let $A$ be a bounded set in $\boldsymbol{R}^{n}$ and let $E \subset$ $\subset L^{\mathbb{1}}\left(\boldsymbol{R}^{n}\right)$ be such that

$$
\begin{array}{ll}
\sup _{f \in E} \int_{\boldsymbol{R}^{n}}|f|<+\infty & \\
\operatorname{spt} f \subset A & \text { for all } f \in E \\
\int_{\boldsymbol{R}^{n}}\left|T_{a} f-f\right| d x \leqq \omega(|a|) & \text { for all } f \in E \\
\lim _{\delta \rightarrow 0} \omega(\delta)=0 &
\end{array}
$$

where $\left(T_{a} f\right)(x)=f(x-a)$, then $E$ is a relatively compact set in $L^{1}\left(\boldsymbol{R}^{n}\right)$.

By using Facts 3.1 and 3.2, it is easy to prove the following lemma.

LemMa 3.3. - Suppose that $f \in L_{1 \mathrm{loc}}^{1}\left(\boldsymbol{R}^{n}\right)$, let $A$ be a bounded set in $\boldsymbol{R}^{n}$ and let $L \subset M\left(\boldsymbol{R}^{n}\right)$ be such that

$$
\begin{aligned}
& \sup _{\lambda \in L}\|\lambda\|<+\infty \\
& \operatorname{spt} \lambda \subset A \quad \text { for all } \lambda \in L
\end{aligned}
$$

then the set $E=\left\{\left.(f * \lambda)\right|_{V} ; \lambda \in L\right\}$ is relatively compact in $L^{1}(V)$ for all bounded rectangles $V \subset \boldsymbol{R}^{n}$.

Here is the key lemma for what follows.

LemMa 3.4. - Let $\boldsymbol{A}$ be an open bounded subset of $\boldsymbol{R}^{n}$ and let us consider the space $M_{n}(A)=\left\{\lambda \in M\left(\boldsymbol{R}^{n}, \boldsymbol{R}^{n^{2}}\right)\right.$ such that $\lambda=\operatorname{rot} T$ for some distribution $T \in \mathbb{D}^{\prime}\left(\boldsymbol{R}^{n}\right)^{n}$ with supt $T \subset \subset A\}$. Then there exists a linear operator

$$
Z: M_{R}(A) \rightarrow L_{100}^{1}\left(\boldsymbol{R}^{n}, \boldsymbol{R}^{n}\right)
$$

suoh that

(i) $\operatorname{rot} Z(\lambda)=\lambda \quad$ in $\boldsymbol{R}^{n}$, for all $\lambda \in M_{R}(A)$;

(ii) the map $\left.\lambda \rightarrow Z(\lambda)\right|_{V}$ is a completely continuous operator $M_{R}(A) \rightarrow L^{1}\left(V, \boldsymbol{R}^{n}\right)$, for any bounded rectangle $\nabla \subset \boldsymbol{R}^{n}$. 
Proof. - Let us consider the kernels

$$
E_{j}(x)=\frac{1}{\alpha_{n}} \frac{x_{j}}{|x|^{n}}=\frac{\partial}{\partial x_{j}} G(x), \quad 1 \leqq j \leqq n,
$$

where $\alpha_{n}$ is the $(n-1)$-dimensional measure of the unit sphere in $\boldsymbol{R}^{n}$ and

$$
G(x)= \begin{cases}-\frac{1}{n-2} \frac{1}{\alpha_{n}} \frac{1}{|x|^{n-2}} & \text { if } n \geqq 3 \\ -\frac{1}{\alpha_{2}} \ln \left(\frac{1}{|x|}\right) & \text { if } n=2\end{cases}
$$

is the fundamental solution of the Laplace equation, i.e.

$$
\sum_{j=1}^{n} \frac{\partial E_{j}}{\partial x_{j}}=\Delta G=\delta
$$

For every $\lambda \in M_{R}(A)$ we consider the function $z=Z(\lambda) \in L_{100}^{1}\left(\boldsymbol{R}^{n}, \boldsymbol{R}^{n}\right)$ defined by

$$
z_{i}=\sum_{j=1}^{n} \lambda_{i j} * E_{j}
$$

If $T \in \mathcal{E}^{\prime}(A)^{n}$ is such that $\operatorname{rot} T=\lambda$ we have, in the sense of distributions,

$$
\begin{aligned}
(\operatorname{rot} z)_{i j}=\sum_{k=1}^{n} \frac{\partial}{\partial x_{j}}\left\{\left[\frac{\partial T_{i}}{\partial x_{k}}-\frac{\partial T_{k}}{\partial x_{i}}\right] * E_{k}\right\}-\frac{\partial}{\partial x_{i}}\left\{\left[\frac{\partial T_{i}}{\partial x_{k}}-\frac{\partial T_{k}}{\partial x_{j}}\right] * E_{k}\right\}= \\
=\left[\frac{\partial T_{i}}{\partial x_{j}}-\frac{\partial T_{j}}{\partial x_{i}}\right] * \sum_{k=1}^{n} \frac{\partial E_{k}}{\partial x_{k}}=\lambda_{i j}
\end{aligned}
$$

and (i) is proved.

Using Lemma 3.3 one gets immediately (ii). q.e.d.

LEMMa 3.5. - For every measure $\mu \in M_{0}\left(\boldsymbol{R}^{n}, \boldsymbol{R}^{n}\right)$ such that rot $\mu \in M\left(\boldsymbol{R}^{n}, \boldsymbol{R}^{n^{2}}\right)$ there exist a function $f \in L_{100}^{1}\left(\boldsymbol{R}^{n}, \boldsymbol{R}^{n}\right)$ and a function $u \in B V_{10 \mathrm{c}}\left(\boldsymbol{R}^{n}\right)$ such that

$$
\mu=D u+f \quad \text { in } \boldsymbol{R}^{n} .
$$

Proof. - If $\mu \in M_{0}\left(\boldsymbol{R}^{n}, \boldsymbol{R}^{n}\right)$, then, by lemma 3.4 , we can consider the function $f=Z(\operatorname{rot} \mu) \in L_{\mathrm{loc}}^{1}\left(\boldsymbol{R}^{n}, \boldsymbol{R}^{n}\right)$ and we have

$$
\operatorname{rot}(\mu-f)=0 \quad \text { in } \boldsymbol{R}^{n}
$$

hence there exists [11] a distribution $u \in D^{\prime}\left(\boldsymbol{R}^{n}\right)$ such that $\mu-f=D u$. On the other hand $\mu-f$ is a measure and it follows [11] that $u \in B V_{\text {Ioc }}\left(\boldsymbol{R}^{n}\right)$. q.e.d. 
Definitron 3.6. - For every measure $\mu \in M_{0}\left(\boldsymbol{R}^{n}, \boldsymbol{R}^{n}\right)$ such that $\operatorname{rot} \mu \in M\left(\boldsymbol{R}^{n}, \boldsymbol{R}^{n^{2}}\right)$ and for every vector field $\psi \in X\left(\boldsymbol{R}^{n}\right)_{n}$, we define the measure $(\psi, \mu) \in \boldsymbol{M}\left(\boldsymbol{R}^{n}\right)$ as

$$
\langle(\psi, \mu), \varphi\rangle=\langle(\psi, D u), \varphi\rangle+\int_{\Omega} f \varphi d x, \quad \varphi \in C_{0}^{\infty}\left(\boldsymbol{R}^{n}\right)
$$

where

$$
\mu=f+D u, \quad f \in L_{10 \mathrm{c}}^{1}\left(\boldsymbol{R}^{n}, \boldsymbol{R}^{n}\right), \quad u \in B V_{10 \mathrm{c}}\left(\boldsymbol{R}^{n}\right)
$$

We remark that definition 3.6 is valid, because for every measure $\mu$ whose curl is a measure there exists (lemma 3.5) at least a pair $f, u$ that satisfies (3.1); moreover, the definition is easily seen to be independent of the choice of $f, u$.

Now we shall define the pairing $(\psi, \mu)$ without the assumption on the support of $\mu$.

Definition 3.7. - Let $\Omega$ be an open set in $\boldsymbol{R}^{n}$ and suppose that $\psi \in X(\Omega)_{n}, \mu \in$ $\in M\left(\Omega, \boldsymbol{R}^{n}\right)$, rot $\mu \in M\left(\Omega, \boldsymbol{R}^{n^{2}}\right)$. For all open sets $A \subset \subset$ cc $\Omega$ choose a function $g \in C_{0}^{\infty}(\Omega)$ such that $g \equiv 1$ on $A$ and consider the distribution

$$
T_{4}=\left.(\psi, g \mu)\right|_{\Delta}
$$

where $(\psi, g \mu)$ is defined in definition 3.6. It is easy to see that if $A_{1}, A_{2}$ are such that $A_{1} \cap A_{2} \neq \emptyset$ one has

$$
\left.T_{\boldsymbol{A}_{1}}\right|_{\boldsymbol{A}_{1} \cup \boldsymbol{A}_{2}}=T_{\boldsymbol{A}_{1} \cup \boldsymbol{A}_{2}}=\left.T_{\boldsymbol{A}_{2}}\right|_{\Lambda_{1} \cup \boldsymbol{A}_{2}}
$$

and by a well known glueing principle [11], there exists one and only one measure in $\Omega$, that we shall denote $(\psi, \mu)$, such that $\left.(\psi, \mu)\right|_{A}=T_{A}$ for all $A \subset \subset \Omega$.

Now we collect a few properties of $(\psi, \mu)$.

THEOREM 3.8. - (i) The map that takes $\psi, \mu$ to $(\psi, \mu)$ is bilinear. (ii) The measure $(\psi, \mu)$ is absolutely continuous with respect to the measure $|\mu|$ and one has precisely, for all Borel sets $B \subset \Omega$,

$$
\int_{B}|(\psi, \mu)| \leqq\|\psi\|_{\infty, \Omega} \int_{B}|\mu|
$$

(iii), For all functions $g \in C^{1}(\Omega)$ with $\sup _{\Omega}(|g|+|D g|)<+\infty$, one has

$$
(\psi, g \mu)=(g \psi, \mu)=(\psi, \mu) g \text {. }
$$


Moreover, if we consider the function $\theta(\psi, \mu, x): \Omega \rightarrow \boldsymbol{R}$ such that

$$
\int_{B}(\psi, \mu)=\int_{B} \theta(\psi, \mu, x)|\mu| \quad \text { for all Borel sets } B \subset \Omega
$$

we have also

(iv) $0(\psi, \mu, x)=\psi(x) \cdot \frac{d \mu}{d|\mu|}(x), \quad|\mu|^{a}$-a.e. in $\Omega$;

(v) $O(\psi, \mu, x)=\theta\left(\psi, \mu_{1}, x\right)|\mu|^{s}$-a.e., if $\mu^{s}=\mu_{1}^{s}$;

(vi) $\theta(\psi, g \mu, x)=\theta(\psi, \mu, x) \operatorname{segn} g(x) \quad|g||\mu|$-a.e. in $\Omega$

$$
\theta(g \psi, \mu, x)=g(x) \theta(\psi, \mu, x) \quad|\mu| \text {-a.e. in } \Omega
$$

if $g \in C^{1}(\Omega) \quad$ and $\quad \sup _{\Omega}(|g|+|D g|)<+\infty$.

Proof. - (i) is obvious. To prove (ii) it is sufficient to show that (3.2) holds for all Borel sets $B \subset A \subset \subset \Omega$. To do that, we can write $\mu=f+D u$ in $A$, for suitable $f$ and $u$, and we have $\mu^{a}=f+(D u)^{a}, \mu^{s}=(D u)^{s}$ and

$$
(\psi, \mu)=\theta(\psi, D u, x)|D u|^{s}+\left((D u)^{a}(x)+f(x)\right) \cdot \psi(x) d x \quad \text { in } A
$$

hence we get

$$
\int_{B}|(\psi, \mu)| \leqq\|\psi\|_{\infty, 4}\left\{\int_{B}|D u|^{s}+\int_{B}|(D u)|{ }^{a}(x)+f(x) d x\right\}=\|\psi\|_{\infty, A} \int_{B}|\mu|
$$

and (ii) is proved. To show (iii), we take a function $\varphi \in C_{0}(\Omega)$, then we write $\mu=$ $=D u+f$ on the support of $\varphi$ and we have

$$
\begin{aligned}
\langle(\psi, g u\rangle, \varphi\rangle=-\int u g \operatorname{div} \psi \varphi d x-\int u g \psi \nabla \varphi d x+\int f g \psi \varphi d x-\int u D g \psi \varphi d x & = \\
=\langle(g \psi, \mu), \varphi\rangle & =\langle(\psi, \mu), g \varphi\rangle
\end{aligned}
$$

which proves (iii). To show (iv), again we take $A$ cc $\Omega$ and write $\mu=f+D u$ in $A$ so that (3.3) holds. On the other hand, we have by definition

$$
(\psi, \mu)=\theta(\psi, \mu, x)|\mu|=\theta(\psi, \mu, x)|D u|^{s}+\theta(\psi, \mu, x)\left|(D u)^{a}(x)+f(x)\right| d x
$$

and, equating the regular parts of the measures on the right sides of $(3.3),(3.4)$, we obtain that (iv) holds $|\mu|^{a}$-a.e. in $A$. Varying the set $A$, (iv) follows. Finally, (v) and (vi) are proved by similar methods; we omit the details. q.e.d. 


\section{4. - Compensated compactness for the pairing $(\psi, \mu)$.}

As a general reference for compensated compactness, we give [12].

We have the following compensated compactness result.

THEOREM 4.1. - Let $\psi_{j}, \psi, \mu_{j}, \mu$ be such that $\psi, \psi_{j} \in X(\Omega)_{n} ; \mu, \mu_{j} \in M\left(\Omega, \boldsymbol{R}^{n}\right)$; $\operatorname{rot} \mu, \operatorname{rot} \mu_{j} \in M\left(\Omega, \boldsymbol{R}^{n}\right)$ and assume that

$$
\begin{gathered}
\psi_{j} \rightarrow \psi \text { in } L^{\infty}(\Omega)-\text { weak } k^{*} \\
\left\|\psi_{j}\right\|_{\infty, \Omega}+\| \text { div } \psi_{j} \|_{L^{n+8}(\Omega)} \leqq C_{1} \quad \text { for all } j \text { for some fixed } \delta>0 \\
\mu_{j} \rightarrow \mu \quad \text { weakly in } M\left(\Omega, \boldsymbol{R}^{n}\right) \\
\left\|\mu_{j}\right\|+\left\|\operatorname{rot} \mu_{j}\right\| \leqq c_{2} \quad \text { for all } j
\end{gathered}
$$

then one has also

$$
\left(\psi_{i}, \mu_{j}\right) \rightarrow(\psi, \mu) \quad \text { weakly in } M(\Omega)
$$

Proof. - It is sufficient to show that for all $\varphi \in C_{0}^{\infty}(\Omega)$ one has

$$
\left\langle\left(\psi_{j}, \mu_{j}\right), \varphi\right\rangle \rightarrow\langle(\psi, \mu), \varphi\rangle
$$

in fact, as, for all $j$, we have

$$
\int_{\Omega}\left|\left(\psi_{j}, \mu_{j}\right)\right| \leqq\left\|\psi_{j}\right\|_{\infty, \Omega}\left|\mu_{\Omega}\right| \leqq c_{1} c_{2}
$$

the convergence (4.1) holds then also for all $\varphi \in O_{0}^{0}(\Omega)$.

Let $p \in C_{0}^{\infty}(\boldsymbol{R})$ be fixed and let $g \in C_{0}^{1}(\Omega)$ be such that $g \equiv 1$ on the support of $\varphi$, then consider the measures $\tilde{\mu}, \tilde{\mu}_{j} \in M_{0}\left(\boldsymbol{R}^{n}, \boldsymbol{R}^{n}\right)$ defined by

$$
\tilde{\mu}=g \mu, \quad \tilde{\mu}_{j}=g \mu_{j} .
$$

We still have $\operatorname{rot} \tilde{\mu}, \operatorname{rot} \tilde{\mu}_{j} \in M\left(\boldsymbol{R}^{n}, \boldsymbol{R}^{\mathbf{n}^{\mathrm{z}}}\right)$ and (4.1) is equivalent to

$$
\left\langle\left(\psi_{j}, \tilde{\mu}_{j}\right), \varphi\right\rangle \rightarrow\langle(\varphi, \mu), \varphi\rangle \text {. }
$$

To prove (4.2) we shall show that for any increasing sequence $j_{k}$ there exists a subsequence $j_{k_{r}}$ such that

$$
\left\langle\left(\psi_{j_{k r}}, \tilde{\mu}_{j_{k_{r}}}\right), \varphi\right\rangle \rightarrow\langle(\psi, \mu), \varphi\rangle
$$


For all $j$ we set $f_{j}=Z\left(\operatorname{rot} \tilde{\mu}_{j}\right) \in L_{100}^{1}\left(\boldsymbol{R}^{n}\right)$, where $Z$ is the operator defined in lemma 3.4, and, as in lemma 3.5, we have

$$
\tilde{\mu}_{j}=f_{j}+D u_{j}
$$

where $u_{j} \in B V_{\text {loe }}\left(\boldsymbol{R}^{n}\right)$, and we may assume that $\int_{Q} u_{j} d x=0$ for all $j$, where $Q$ is some fixed eube containing the support of $g$. As the norms $\left\|\operatorname{rot} \tilde{\mu}_{j}\right\|$ are bounded, the sequence $f_{j}$ is bounded and relatively compact in $L^{1}\left(Q, \boldsymbol{R}^{n}\right)$ (by lemma 3.4). As the norms $\left\|\mu_{j}\right\|$ and $\left\|f_{j}\right\|_{L^{1}(Q)}$ are bounded and $\int_{Q} u_{j} d x=0$, we have that the sequence $u_{j}$ is bounded in $B V(Q)$. We conclude that for any increasing sequence $j_{k} \in N$ there exists a subsequence $j_{k_{r}}$ and two functions $f \in L^{1}(Q), u \in B V(Q)$ such that

$$
\begin{array}{ll}
f_{j_{k_{r}}} \rightarrow f & \text { in } L^{1}(Q) \\
u_{j_{k_{r}}} \rightarrow u & \text { in } L^{p}(Q), \text { where } \frac{1}{p}+\frac{1}{n+\delta}=1 \\
\operatorname{rot} f=\operatorname{rot} \tilde{\mu}, & \tilde{\mu}=D u+f .
\end{array}
$$

To conclude, we have that

$$
\begin{aligned}
\left\langle\left(\psi_{j_{k_{r}}}, \mu_{j_{k_{r}}}\right), \varphi\right\rangle=-\int_{\Omega} & u_{j_{k_{r}}} \psi_{j_{k_{r}}} D \varphi d x-\int_{\Omega} u_{j_{k_{r}}} \operatorname{div} \psi_{j_{k_{r}}} \varphi d x+\int_{\Omega} f_{j_{k_{r}}} \varphi d x \rightarrow \\
& \rightarrow-\int_{\Omega} u \psi D \varphi d x-\int_{\Omega} u \operatorname{div} \psi \varphi d x+\int_{\Omega} f \varphi d x=\langle(\psi, \tilde{\mu}), \varphi\rangle \text {. q.e.d. }
\end{aligned}
$$

Under the hypotheses of theorem 4.1 , the integrals $\int_{\Omega}\left(\psi_{j}, \mu_{j}\right)$ need not converge to $\int_{\Omega}(\psi, \mu)$. To ensure that, one needs the supplementary assumption $\int_{\Omega}\left|\mu_{j}\right| \rightarrow \int_{\Omega}|\mu|$, as it is shown in the next theorem.

THEOREM 4.2. - Let $\mu, \mu_{j}, \psi, \psi_{j}$ be as in theorem 4.1, and assume moreover that

then one has also

$$
\int_{\Omega}|\mu| \rightarrow \int_{\Omega}|\mu|
$$

$$
\int_{\Omega}\left(\psi_{i}, \mu_{j}\right) \varphi \rightarrow \int_{\Omega}(\psi, \mu) \varphi
$$

for all $\varphi \in C^{0}(\Omega) \cap L^{\infty}(\Omega)$.

Proof. - Take a fixed function $\varphi \in C^{0}(\Omega) \cap L^{\infty}(\Omega)$ and let $\varepsilon>0$ be given. There exists a number $\delta=\delta(\varepsilon)>0$ such that

$$
\int_{\Omega \Omega_{s}}|\mu|<\varepsilon
$$


where $\Omega_{\delta}=\{x \in \Omega \mid$ dist $(x, \partial \Omega)>\delta\} . \Delta$ s $\mu_{j} \rightarrow \mu$ weakly, we have

$$
\min _{j \rightarrow \infty} \int_{\Omega_{\delta}}|\mu| \geqq \int_{\Omega_{\delta}}|\mu|
$$

and, recalling (4.3), we get

$$
\max _{j \rightarrow \infty} \lim _{\Omega \backslash \Omega_{0}}|\mu| \leqq \int_{\Omega \backslash \Omega_{0}}|\mu|<\varepsilon .
$$

Now, we take a function $\eta \in C_{0}^{0}(\Omega)$ such that $\eta \equiv 1$ on $\Omega_{\delta}$ and we write

$$
\begin{aligned}
\int_{\Omega}\left(\psi_{j}, \mu_{j}\right) \varphi & -\int_{\Omega}(\psi, \mu) \varphi= \\
= & {\left[\int_{\Omega}\left(\psi_{j}, \mu_{j}\right) \varphi \eta-\int_{\Omega}(\psi, \mu) \varphi \eta\right]+\left[\int_{\Omega}\left(\psi_{j}, \mu_{j}\right) \varphi(1-\eta)-\int_{\Omega}(\psi, \mu) \varphi(1-\eta)\right] }
\end{aligned}
$$

where the first term in brackets goes to zero, because of theorem 4.1, and the second terms in brackets, for $j$ sufficiently big, is bounded by

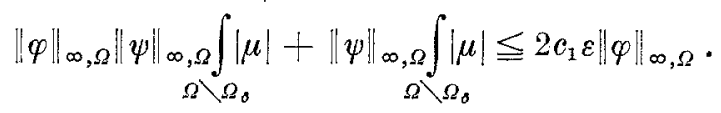

Taking the limit in (4.4) for $j \rightarrow \infty$ we get our result, as $\varepsilon>0$ is arbitrary. q.e.d.

\section{5. - Appendix.}

LeMrMA 5.1. - Let $\Omega$ be any open set in $\boldsymbol{R}^{n}$, let $u \in B V(\Omega)$ be fixed and set $u_{j}=\tilde{\mu} * \eta_{j}$, where

$$
\tilde{\mu}(x)= \begin{cases}u(x) & \text { if } x \in \Omega \\ 0 & \text { if } x \notin \Omega\end{cases}
$$

and $\eta_{j} \in C_{0}^{\infty}\left(\boldsymbol{R}^{n}\right)$ is a sequence of mollifiers. Then one has

$$
u_{j} \rightarrow u \quad \text { in } L^{1}(\Omega) .
$$

If $A$ is an open set, $A \subset \subset, \Omega$, one has also

$$
\begin{aligned}
& \int_{\Delta}\left|D u_{j}\right| \rightarrow \int_{\Delta}|D u| \quad \text { if } \int_{\partial \Delta}|D u|=0 \\
& \int_{\boldsymbol{A}}|D u-h(x) d x| \leqq \min _{j \rightarrow \infty} \lim _{\boldsymbol{A}}\left|D u_{j}-h\right| d x \leqq \max _{j \rightarrow \infty} \lim \int_{\boldsymbol{A}}\left|D u_{j}-h\right| d x \leqq \\
& \leqq \int_{\frac{A}{A}}|D u-h d x| \quad \text { for all } h \in L^{1}(\Omega) \text {. }
\end{aligned}
$$




\section{Moreover:}

$$
\begin{aligned}
& \text { if } u \in B V(\Omega) \cap L^{g}(\Omega), q<+\infty \text {, one has also } u_{j} \rightarrow u \text { in } L^{q}(\Omega) \\
& \text { if } u \in B V(\Omega) \cap L^{\infty}(\Omega) \text {, one has }\left\|u_{j}\right\|_{\infty, \Omega} \leqq\|u\|_{\infty, \Omega} u_{j} \rightarrow u \text { in } L^{\infty}(\Omega) \text {-veak } *^{*} \\
& \text { if } u \in B V(\Omega) \cap L^{\infty}(\Omega) \cap C^{0}(\Omega), \text { one has also } u_{j} \rightarrow u \text { in } C_{\mathrm{loc}}^{0}(\Omega) .
\end{aligned}
$$

Proof. - (5.1), (5.4), (5.5), (5.6) are standard and (5.2) follows from (5.3). To prove $(5.3)$, we notice that

$$
D u_{j}=(D u) * \eta_{j}=(D u)^{a} * \eta_{j}+(D u)^{s} * \eta_{j}
$$

where

$$
(D u)^{a} * \eta_{j} \rightarrow(D u)^{a} \quad \text { in } L^{1}(\Omega)
$$

hence

$$
\begin{aligned}
\max _{j \rightarrow \infty} \lim _{\boldsymbol{A}}\left|D u_{j}-h\right| d x & \leqq \lim _{j \rightarrow \infty} \int_{\boldsymbol{A}}\left|(D u)^{a} * \eta_{j}-h\right| d x+ \\
& +\max _{j \rightarrow \infty} \lim _{\boldsymbol{A}}\left|(D u)^{s} * \eta_{j}\right| \leqq \int_{\boldsymbol{A}}\left|(D u)^{a}-h\right| d x+\int_{\frac{A}{A}}|D u|^{s}=\int_{\frac{A}{A}}|D u-h| .
\end{aligned}
$$

On the other hand, we bave $\left(D u_{j}-h\right) \rightarrow(D u-h)$, and, because of the semicontinuity of the total variation, (5.3) is proved. q.e.d.

LEMMA 5.2. - Let $\Omega$ be any open set in $\boldsymbol{R}^{n}$ and let $u \in B V(\Omega)$ be fixed. Then there exists a sequence of functions $u_{j} \in C^{\infty}(\Omega) \cap \bar{B} V(\Omega)$ such that

$$
\begin{aligned}
& u_{j} \rightarrow u \quad \text { in } L^{1}(\Omega) \\
& \int_{\Omega}\left|D u_{j}\right| \rightarrow \int_{\Omega}|D u| \\
& \max _{j \rightarrow \infty} \lim _{\Delta}\left|D u_{j}\right| \leqq \int_{-}|D u| \quad \text { for all open sets } A \subset \subset \Omega \\
& \int_{\Omega}\left|D u_{j}-h\right| d x \rightarrow \int_{\Omega}|D u-h d x| \quad \text { for all } h \in L^{1}(\Omega) .
\end{aligned}
$$

Moreover:

(5.11) if $u \in B V(\Omega) \cap L^{a}(\Omega), q<+\infty$, one can find the functions $u_{j}$ such that $u_{j} \in L^{\alpha}(\Omega), u_{j} \rightarrow u$ in $L^{q}(\Omega)$ 
GaBRIELE ANZELLotT: Pairings between measures and bounded, etc. $\quad 317$

(5.12) if $u \in B V(\Omega) \cap L^{\infty}(\Omega)$, one can find the $u_{j}$ such that $\left\|u_{j}\right\|_{\infty, \Omega} \leqq\|u\|_{\infty, \Omega}$ and $u_{j} \rightarrow u$ in $L^{\infty}(\Omega)-w e a k^{*}$

(5.13) if $u \in B V(\Omega) \cap L^{\infty}(\Omega) \cap C^{0}(\Omega)$ one can find the $u_{j}$ such that $u_{j} \rightarrow u$ in $C_{\mathrm{loc}}^{0}(\Omega)$ also holds.

Finally:

(5.14) if $\partial \Omega$ is Lipschitz continuous one can find the $u_{j}$ such that

$$
\left.u_{j}\right|_{\partial \Omega}=\left.u\right|_{\partial \Omega} \quad \text { for all } j
$$

Proof. - (5.7) and (5.8) are proved in [4]; (5.11), (5.12), (5.13) follow easily by the same proof; (5.14) is proved in [7] and (5.9), (5.10) follow easily by adapting to the proof in [4] the argument given in the proof of lemma 5.1.

Corollary 5.3. - If we take $h=(D u)^{a}$ in $(5.10)$ we get

$$
\int_{\Omega}\left|D u_{j}-(D u)^{a}\right| \rightarrow \int_{\Omega}|D u|^{s} .
$$

LEMMA 5.4. - Assume that $\partial \Omega$ is Lipschitz continuous. If $u \in H^{1,1}(\Omega) \cap L^{\infty}(\Omega) \cap$ $\cap C^{\circ}(\Omega)$ and $\left.u\right|_{\partial \Omega}=0$, then there exists a sequence of functions $g_{j} \in C_{0}^{\infty}(\Omega)$ such that

$$
\begin{array}{ll}
g_{i} \rightarrow u & \text { in } H^{1,1}(\Omega) \\
g_{j} \rightarrow u & \text { in } C_{\mathrm{loc}}^{0}(\Omega) \\
\left\|g_{j}\right\|_{\infty, \Omega} \leqq\|u\|_{\infty, \Omega} & \text { for all } j .
\end{array}
$$

The proof of lemma 5.4 can be obtained by standard techniques in Sobolev space theory.

LEMMA 5.5. - Let $\Omega$ be a bounded open set in $\boldsymbol{R}^{n}$ with a Lipsehitz boundary. Then, for any given function $u \in L^{1}(\partial \Omega)$ and for any given $\varepsilon>0$ there exists a function $w \in H^{1,1}(\Omega) \cap C^{0}(\Omega)$ such that

$$
\begin{aligned}
& \left.w\right|_{\partial \Omega}=u \\
& \int_{\Omega}|D w| \leqq \int_{\partial \Omega}|u|+\varepsilon \\
& w(x)=0 \quad \text { if } \operatorname{dist}(x, \partial \Omega)>\varepsilon .
\end{aligned}
$$

Moreover, for any fixed number $q \geqq 1, q<+\infty$, one can find the function $w$ such that

$$
\|w\|_{L^{q}(\Omega)} \leqq \varepsilon
$$


Finally, if one has also $u \in L^{\infty}(\Omega)$, one can find w such that

$$
\|w\|_{\infty, \Omega} \leqq\|u\|_{\infty, \partial \Omega} .
$$

The proof of Lemma 5.5 is easily obtained by the same technique that GAGLIARDo [6] uses in proving his extension theorem $L^{1}(\partial \Omega) \rightarrow H^{1,1}(\Omega)$.

\section{REFERENCES}

[1] G. AnzellotTi, On the existence of the rates of stress and displacement for Prandtl-Reuss plastieity, Quaterly of Appl. Math., July 1983.

$\left[2^{7}\right.$ G. Anzeliotsi, On the extremal stress and displacement in Hencky plasticity, Duke Math. J., March 1984.

[3] G. ANZELIOTT, On the minima of funetionals with linear growth, to appear.

[4] G. Anzeliotti - M. Graquinta, Funzioni BV e tracce, Rend. Sem. Mat. Padova, 60 (1978), pp. 1-21.

[5] H. FEDERer, Geometric measure theory, Springer-Verlag (1969).

[6] E. GaGLiando, Oaratterizzazione delle tracce sulla frontiera relative ad alcune classi di funzioni in $n$ variabili, Rend. Sem. Mat. Padova, 27 (1957), pp. 284-305.

[7] E. Giusti, Minimal surfaces and functions of bounded variation, Notes on Pure Math. Australian National University, Canberra (1977).

[8] R. KoHN - R. Temam, Dual spaces of stresses and strains, with application to Hencky plasticity, to appear.

[9] M. Muranda, Superfici cartesiane generalizzate ed insiemi di perimetro finito sui prodotti cartesiani, Ann. Scuola Normale Sup. Pisa, S. III, 18 (1964), pp. 513-542.

[10] F. MuraT, Compacité par compensation, Ann. Scuola Normale Sup. Pisa, S. IV, 5 (1978).

[11] L. Sohwartz, Théorie des distributions, Hermann (1957, 1959).

[12] L. TARTAR, The compensated compactness method applied to systems of conservation laws, in "Systems of Nonlinear partial differential equations", J. M. Ball (ed.), Reidel Publishing Co. (1983).

[13] R. Temam, Navier-Stokes Equation, North Holland, Amsterdam (1977). 